ORIGINAL ARTICLE

\title{
Odontoid lateral mass asymmetry: do we over-investigate?
}

\author{
J A Harty, B Lenehan, S K O'Rourke
}

Emerg Med J 2005;22:625-627. doi: 10.1136/emj.2003.014100

\begin{abstract}
See end of article for authors' affiliations

Correspondence to: J A Harty, The Mews, 61 Serpentine Ave Ballsbridge, Dublin 4 Ireland; jaharty@hotmail. com

Accepted for publication 1 September 2004
\end{abstract}

\begin{abstract}
Objectives: This study aimed to evaluate the necessity for further radiological investigation in patients with suspected traumatic rotatory subluxation of the atlanto-axial complex on plain radiography following acute cervical trauma and outline guidelines for assessment of patients with atlanto-axial asymmetry on plain radiography.

Methods: A retrospective review of all patients who had undergone atlanto-axial CT scanning as a result of radiographic $\mathrm{C} 1-\mathrm{C} 2$ asymmetry following cervical spine trauma. The plain $x$ ray and $\mathrm{CT}$ images were reviewed retrospectively and correlated with the clinical presentation and outcome.

Results and conclusion: Records of 29 patients (16 men, 13 women; age range 21-44 years) were reviewed. All patients were found to have atlanto-odontoid asymmetry on the initial plain $x$ ray. CT images of none of the patients revealed rotatory subluxation. Ten patients (32\%) were found to have congenital odontoid lateral mass asymmetry. All patients were treated conservatively without any further intervention. On review, in 19 patients the orientation of the $x$ ray beam in combination with head rotation was found to be at fault. Approximately 1050 trauma cervical spine $x$ rays were taken in the department where this study was conducted over the period 1999-2001. This study identified 10 patients out of a total of 29 as having congenital odontoid lateral mass asymmetry. This represents approximately $1 \%$ of the patients attending the emergency department. Thus congenital odontoid lateral mass asymmetry should be considered in the differential diagnosis following acute cervical trauma.
\end{abstract}

A dvanced Trauma Life Support (ATLS) has resulted in a significant increase in the number of radiological examinations, and thus an associated increase in the number of suspected cases of traumatic atlanto-axial rotatory fixation (AARF). Rotatory fixation of the cervical spine is a rare but serious condition in the adult that presents with torticollis or "cock-robin position", which is irreducible. It is often associated with arthritides, infection, or Grisel's syndrome in children but is rarely associated with trauma. The diagnosis is a clinical one with torticollis, high neck pain, and no neurological abnormality. Plain $x$ rays should include a "peg view" and a lateral cervical spine. Traumatic AARF is suspected radiologically when odontoid lateral mass asymmetry is present in the open mouth view. The increase in the suspected diagnosis on plain $x$ rays has led to a parallel increase in the number of computed tomography (CT) scans of $\mathrm{C} 1-\mathrm{C} 2$ taken to diagnose AARF.

The aim of our study was to retrospectively evaluate the imaging appearances in a series of adult patients who underwent cervical spine CT after atlanto-axial asymmetry was shown on radiography. We then assessed the clinical outcome of this group of patients and established a protocol for appropriate investigation of atlanto-axial asymmetry.

\section{MATERIALS AND METHODS}

We retrospectively reviewed CT records of adult patients with a history of acute neck trauma from the period 1999-2001 at our institution. All patients had been referred through our Accident and Emergency (A\&E) Department. None of these patients had any other injuries apart from the suspected neck injury. Atlanto-axial asymmetry was suspected on the basis of a standard cervical $x$ ray series showing evidence of odontoid lateral mass asymmetry. There was no evidence of an increase in the atlanto-dens interval on the lateral view in any patient. All patients went on to have a CT scan of C1-C3 with $2 \mathrm{~mm}$ axial cuts from the occiput to C3. Seven patients underwent dynamic CT scanning.

\section{RESULTS}

We included a total of 29 patients ( 16 men, 13 women; age range 21-44 years) in this study. The mechanisms of injury were: road traffic accident $(n=21)$, sports related $(n=3)$, and falls $(n=5)$. In $10 / 29$ patients at least one further attempt was made to take an open mouth view prior to CT. The sources of CT referral were: A\&E $(n=9)$, orthopaedics $(n=9)$, and radiology $(n=11)$. Fifteen patients $(52 \%)$ were scanned during on-call hours. None of the patients had torticollis or neurological deficit. A review of the records revealed that only $4 / 29$ patients had cervical tenderness in the $\mathrm{C} 1-\mathrm{C} 2$ region. All patients were treated conservatively. Seven patients $(25 \%)$ were diagnosed as having a minor degree of "rotation" $\left(<10^{\circ}\right)$ on CT. Dynamic CT in these patients showed correction of any rotatory asymmetry. Follow up radiography showed persisting C1-C2 asymmetry in 10/29 patients. CT scan findings confirmed congenital atlanto-axial asymmetry in this group.

We carried out an audit of the number of cervical spine $x$ rays taken per annum in the trauma setting in our department, over the same period 1999-2001; 1050 cervical spine films were taken during this period. All plain $x$ rays in

Box 1: Fielding and Hawkins' classification of atlanto-axial rotatory fixation

- Type I: Rotatory fixation without anterior displacement of the atlas

- Type II: Rotatory fixation with anterior displacement of the atlas by $3-5 \mathrm{~mm}$

- Type III: Rotatory fixation with anterior displacement of more than $5 \mathrm{~mm}$

- Type IV: Rotatory fixation with posterior displacement 


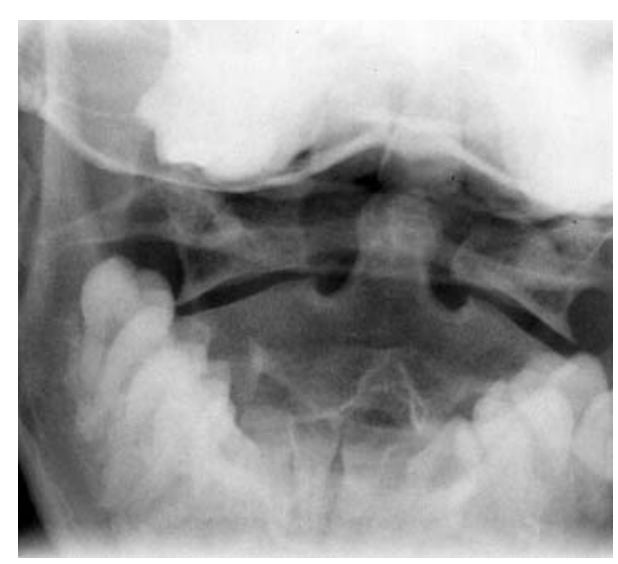

Figure 1 Poorly centred peg view showing odontoid lateral mass asymmetry.

this series were then assessed to determine the underlying reason for asymmetry. In 19 patients, the orientation of the $x$ ray beam in combination with head rotation was found to be at fault. Thus we found 10 patients with congenital atlanto-axial asymmetry, which accounts for approximately $1 \%$ of all films taken during this time period.

\section{DISCUSSION}

Fielding and Hawkins classified AARF into four types (box 1). ${ }^{1}$ Types I and II appear to cause the most controversy when making a diagnosis, and probably account for the vast majority of cases in children. It is a rare traumatic injury in adults. An increase in ATLS management of trauma cases has led to an associated increase in the number of cervical spine series taken in A\&E. Lack of cooperation on the part of the patient or diminished active movement of the neck may render it impossible to take ATLS $x$ rays.

Atlanto-axial asymmetry following neck trauma may be due to a number of factors ranging from technical deficiencies to normal variants to actual AARF. Technical factors include misalignment of the $x$ ray beam in relation to the patient's head. ${ }^{2}$ A number of characteristics of the misaligned beam on the peg view will help determine whether this is the cause of the asymmetry (figs 1 and 2):

- Is the spinous process of C2 central?

- Are the facet joints symmetrical?

- Are the molars, mandible, and incisors symmetrical?

Resnick recommends five open mouth views rotated/tilted left and right. ${ }^{3}$ Odontoid lateral mass asymmetry should persist in all views. ${ }^{3}$ On the lateral view the atlanto-dens interval should be $<3 \mathrm{~mm}$ in an adult and $<5 \mathrm{~mm}$ in a child. This interval should be seen to be increased in the lateral view or to increase in flexion views. ${ }^{4}$

The odontoid process develops from two parallel columns of bone which usually grow symmetrically. ${ }^{5}$ If there is dysplasia of one of these columns, it will give an impression of asymmetry of the odontoid lateral mass space on the affected side. Hypoplasia of the lateral mass of the atlas will have the same effect. ${ }^{6}$ On review of the 10 CT scans that showed persisting asymmetry, we found either mild hypoplasia of odontoid or the lateral mass of the atlas on each occasion (fig 3).

In terms of costs and radiation exposure, proceeding directly to CT without taking repeated plain films has a significant impact on patient welfare and departmental expenditure. As stated above almost $50 \%$ of our patients underwent CT scanning out of hours. We estimate that

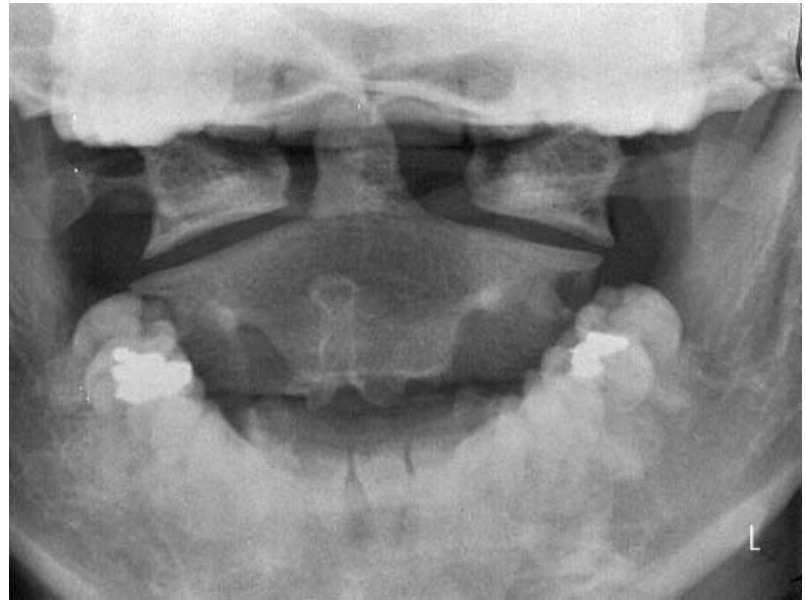

Figure 2 Well centred peg view showing persisting odontoid lateral mass asymmetry.

cervical spine CT scanning exposes a patient to approximately $2000 \mu \mathrm{Sv}$ as opposed to $70 \mu \mathrm{Sv}$ for a plain film. Thus even after allowing for several repeated films to be taken, the radiation exposure is still significantly less than with a CT scan. In our department, a CT scan of the cervical spine has a fixed cost of $€ 450$, as opposed to $€ 60$ for a plain $x$ ray. The cost of repeating several plain films is still less than a CT scan. We have not factored in the associated cost of paying an oncall CT radiographer to attend out of hours.

In summary, we did not find any evidence of clinically significant traumatic atlanto-axial asymmetry in this series. All abnormalities were due to technical factors or were normal variants. Five open mouth views in rotation and lateral flexion may not be safe/advisable for acute trauma, where there is neck pain and significant mechanism of injury, and certainly not if there is any altered sensorium. The very presence of odontoid lateral mass asymmetry on plain film in a trauma patient should raise the suspicion of fracture or dislocation until proved otherwise. A well centred peg view is the next investigation if one is suspicious of atlanto-axial asymmetry. Persistent asymmetry should be seen on a series of five plain $x$ rays before proceeding to CT scan, which should be reserved for torticollis or suspected fracture. Dynamic CT is essential to out rule AARF. ${ }^{6}$ One should be aware that up to $1 \%$ of all cervical spine films in the trauma setting may show asymmetry due to congenital odontoid lateral mass asymmetry. This paper concludes that a traumatic cause for AARF in an adult is not seen without association with a fracture. We did not find any evidence in

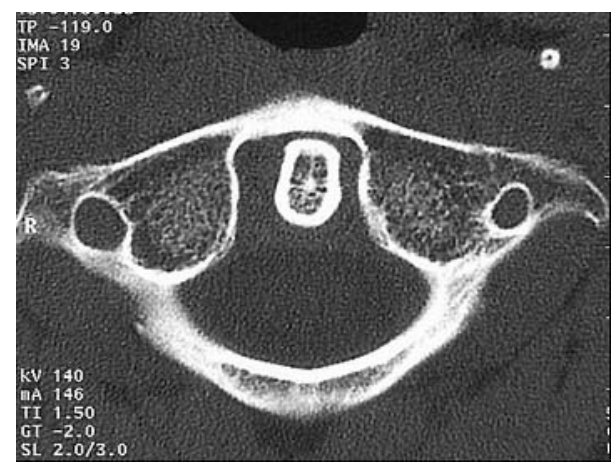

Figure 3 Computed tomography scan showing mild hypoplasia of right lateral mass of atlas and persisting odontoid lateral asymmetry. 
the literature suggesting that AARF occurs in the absence of a fracture in a trauma setting. We found no evidence of fracture on any of our plain films, or of torticollis or neurological deficits, yet CT scanning was done in all these patients.

\section{Authors' affiliations}

J A Harty, B Lenehan, S K O'Rourke, Department of Orthopaedics, St Vincent's University Hospital, Dublin, Ireland

Competing interests: none declared

\section{REFERENCES}

1 Fielding JW, Hawkins RJ. Atlanto-axial rotatory fixation (fixed rotatory subluxation of the atlanto-axial joint). J Bone Joint Surg (Am) 1977;59-A:37-44.

2 Shapiro R, Youngberg AS, Rothman S. The differential diagnosis of traumatic lesions of the occipito-atlanto-axial segment. Radiol Clin N Am 1973;XI:505-26.

3 Resnick D. Bone and Joint Imaging. WB Saunders, 1989:870-1.

4 Mihara H, Onari K, Hachiya M, et al. Follow-up study of conservative treatment for atlantoaxial rotatory displacement. J Spinal Disord 2001;14:494-9.

5 Bailey DK. The normal cervical spine in infants and children. Radiology 1952;59:713.

6 Roche CJ, King SJ, Dangerfield PH, et al. The atlanto-axial joint: physiological range of rotation on MRI and CT. Clin Radiol 2002;57:103-8.

\section{bmjupdates+}

bmjupdates+ is a unique and free alerting service, designed to keep you up to date with the medical literature that is truly important to your practice.

bmjupdates+ will alert you to important new research and will provide you with the best new evidence concerning important advances in health care, tailored to your medical interests and time demands.

\section{Where does the information come from?}

bmiupdates+ applies an expert critical appraisal filter to over 100 top medical journals

A panel of over 2000 physicians find the few 'must read' studies for each area of clinical interest

Sign up to receive your tailored email alerts, searching access and more...

www.bmjupdates.com 